\title{
Detection and Tracking of Coherent Features in a Mach 4 Turbulent Boundary Layer
}

\author{
Ivan Marusic* \\ Graham V. Candler** \\ Victoria Interrante ${ }^{\dagger}$ \\ M. Pino Martin $\ddagger$ \\ Pramod K. Subbareddy $\S$ \\ Alin Moss $§$ \\ University of Minnesota, Minneapolis MN 55455
}

\begin{abstract}
The detection and tracking of coherent vortical structures in a turbulent compressible boundary layer is considered. Several vortex identification methods are evaluated. It is found that for this flow, the discriminant of the characteristic polynomial of the velocity gradient tensor is the most useful. It tends to highlight vortical structures that are located farther from the wall, and the feature detection is less sensitive to the level than the other methods evaluated. The flow fields are visualized using advanced volume rendering methods that allow large datasets to be analyzed efficiently. This methods have also been adapted to volume fill specific structures so that their evolution can be tracked in time. These approaches promise to make the analysis of large DNS datasets more efficient and quantitative.
\end{abstract}

\section{INTRODUCTION}

The direct numerical simulation of turbulent flows is becoming more and more commonplace. These calculations produce vast quantities of 3-D time-dependent data - often at rates much greater than it is possible to analyze the data being generated. For example, using a 128-processor Cray T3E, a DNS produces about 10 terabytes of data per day. There are currently no effective methods to visualize these data, and then to use the information to draw quantative conclusions about physical relationships represented by the data.

This paper is an initial step toward generating a set of tools to efficiently analyze large DNS datasets.

\footnotetext{
* Assistant Professor, Aerospace Engineering \& Mechanics

** Professor, Aerospace Engineering \& Mechanics

$\dagger$ Assistant Professor, Computer Science \& Engineering

$\ddagger$ Post-Doctoral Research Associate, Aerospace Engineering \& Mechanics

$\S$ Graduate Research Assistant, Aerospace Engineering \& Mechanics

Copyright (C) 2000 by Ivan Marusic. Published by the American Institute of Aeronautics and Astronautics, Inc. with permission.
}

Our approach is to focus on the identification of the coherent vortical structures that are the basic components of turbulent flow. We investigate several detection methods and determine which provides the most information about the turbulent motion. This analysis is facilitated with the use of advanced visualization methods that allow volume rendered images to be generated efficiently. More importantly, the visualization methods make it possible to color-fill specific structures to follow their motion through the flow field. Thus, these visualization methods will enable a new and more quantitative understanding of DNS datasets.

\subsection{Details of simulation}

In this paper, we present a preliminary investigation of a large DNS dataset of a Mach 4 turbulent boundary layer. The flow conditions are characteristic of a turbulent supersonic boundary layer at high altitude flight conditions. The edge Mach nunber is 4 , with an edge temperature of $5000 \mathrm{~K}$ and density of $0.5 \mathrm{~kg} / \mathrm{m}^{3}$. The wall is taken to be adiabatic. The Reynolds number based on the momentum thickness is $R_{\theta}=7670$ and the Karman number $R e_{\tau}=\delta u_{\tau} / \nu=700$, where $\delta$ is the boundary layer thickness, $u_{\tau}$ is the wall shear velocity, and $\nu$ is the kinematic viscosity.

The DNS is performed on a grid with $384 \times 128 \times 128$ points in the streamwise, spanwise, and wall-normal directions, respectively. The homogeneous directions are equispaced and the wall-normal direction is exponentially stretched from the wall. The domain size is $8 \delta \times 2 \delta \times 15 \delta$ and the grid resolution is $\Delta x^{+}=$ $\Delta x u_{\tau} / \nu=15, \Delta y^{+}=11$, with $\Delta z^{+}$varying from 0.15 to 46 across the boundary layer (Martin and Candler 2000). The DNS was performed using a high-accuracy, low-dissipation, shock-capturing finitedifference method specifically designed for this type of problem (Weirs and Candler 1997). The time integration uses a parallelizable second-order accurate implicit method (Olejniczak and Candler 1997). The results of the DNS agree very well with theoretical and 
experimental correlations.

We found that an appropriate non-dimensional time between frames of movies for this flow is $t u_{\tau} / \delta=5.6 \times$ $10^{-4}$ or $t U_{e} / \delta=0.013$. This corresponds to visualizing the data every 92 time steps in the simulation.

\subsection{Coherent structure identification}

A number of techniques have been proposed for the identification of a vortex or a coherent vortical structure. The identification process is complicated by the fact that different definitions exist for what constitutes a vortex (Jeong \& Hussain 1995, Chong et al. 1990). In this paper we restrict our attention to three techniques for vortex identification which are associated with the velocity gradient tensor $A_{i j}=\partial u_{j} / \partial u_{i}$. These include looking at level-sets of enstrophy $E$, "swirl strength" $S$ (Zhou et al. 1999) and "discriminant" $\Delta$ (Blackburn et al. 1996). All three have the advantage of being invariant under a Galilean transformation and therefore do not depend on the velocity of the observer. To define these parameters, we first consider the symmetric and anti-symmetric parts of $A_{i j}$

$$
A_{i j}=S_{i j}+\Omega_{i j} .
$$

We also consider the characteristic cubic equation for $A_{i j}$, given by

$$
\lambda^{3}+P \lambda^{2}+Q \lambda+R=0
$$

where the eigenvalues $\lambda$ are the roots of the equation and $P, Q$ and $R$ are the invariants of $A_{i j}$, given by

$$
\begin{gathered}
P=-A_{i i} \\
Q=\frac{1}{2}\left(P^{2}-S_{i j} S_{j i}-\Omega_{i j} \Omega_{j i}\right)
\end{gathered}
$$

and

$$
R=\frac{1}{3}\left(-P^{3}+3 P Q-S_{i j} S_{j k} S_{k i}-3 \Omega_{i j} \Omega_{j k} \Omega_{k i}\right) .
$$

The enstrophy $E$, which is the square of the vorticity vector, is given by

$$
E=-2 \Omega_{i j} \Omega_{j i}
$$

and is seen to make up part of the second invariant $Q$. $\Delta$ is the discriminant of (2) and is given by

$$
\Delta=\frac{\hat{R}^{2}}{4}+\frac{\hat{Q}^{3}}{27}
$$

where $\hat{R}=R+\frac{2}{27} P^{3}-\frac{1}{3} P Q$ and $\hat{Q}=Q-\frac{1}{3} P^{2}$. Chong et al. (1990) used this framework to describe a general classification technique for fluid flow based on the topology of the instantaneous streamlines about a point. When $\Delta$ is positive, the velocity gradient tensor $A_{i j}$ has a real eigenvalue and a pair of conjugated complex eigenvalues

$$
\lambda_{r} ; \quad \lambda_{c r} \pm i \lambda_{c i}
$$

and the local topology is that of a spiraling focus. Zhou et al. (1999) define "swirl strength" $S$ as simply being the imaginary part of the complex eigenvalue pair

$$
S=\lambda_{c i},
$$

and thus implies that $\Delta$ has a non-negative value.

\section{VISUALIZATION TOOLS}

The DNS data are rendered using a classical raycasting volume renderer (Levoy 1988). At each time step, the 3D normalized level-set data are first resampled into a high resolution uniform grid $(1018 \times 251 \times$ 137 ), and then to minimize disk space and memory requirements quantized into 8-bit values spanning the range $0-255$, such that the remapping is uniform across all time steps. Images are created by tracing a bundle of rays from the eye point through the 3D data, such that each ray passes through the center of a pixel in the image plane, and returns the color value to be assigned to that pixel. The final pixel color is obtained by compositing local color and opacity values trilinearly interpolated from the uniform grid to sample points at evenly spaced intervals along the ray. Local color values are computed at each grid point using a simple two-sided Phong illumination model (Foley et al. 1990):

$$
\text { sample_color }=k_{a}+k_{d} \times|N \cdot L|+k_{s} \times|N \cdot H|^{n},
$$

where $k_{a}, k_{d}$ and $k_{s}$ are the ambient, diffuse and specular reflectivities multiplied by the intensity of illuminant, respectively; which together with the exponent $n$ are defined by the user. $N$ is a unit vector in the direction of the data value gradient, which can be interpreted as the unit normal to the level surface at the grid point, $L$ is the direction of the parallel illumination from an infinitely distant light source, and $H$ is the vector halfway between $L$ and the line of sight, or direction of projection. Local opacity values are defined at each grid point as a gradient magnitudeweighted linear function of the input data values according to a user-specified data-to-opacity correspondence given at key breakpoints. The volume rendering approach in effect finesses the definition of the apparent surfaces, allowing us greater flexibility to portray the essential structures of interest in a form that is more robust than isosurface rendering to the local 
effects of isolated samples and minor fluctuations or nonuniformities in the distribution of the chosen levelset.

\section{RESULTS AND DISCUSSION}

Figures 1, 2 and 3 show visualizations for the levelset results for $E, \Delta$ and $S$ respectively for one time step in the simulation. Figures 1(a), (b) and (c) correspond to descending thresholds of $E$. The highest levels of enstrophy are observed near the wall, and as seen in Fig. 1(a) the features become smeared in this region and it is difficult to distinguish different structures. The discriminant results in Fig. 2 show a dramatically different picture with much better defined coherent regions in the near-wall regions. This is consistent with the incompressible channel flow results of Blackburn et al. (1996), where level-sets of $\Delta$ are seen to extract clear features in regions where level-sets of $E$ appear saturated. The results in Fig. 2 are for a low level of $\Delta$ above zero and the results were found to be much less sensitive to the actual threshold level as compared to $E$. The swirl results shown in Fig. 3 reveal a qualitative picture which is similar to the results of $\Delta$ and $E$ with many more features shown in the outer flow region compared to $\Delta$.

Regular events are observed, which are perhaps best shown in in Figs. 2(c) and 3(c). These are vortex structures lifted up from the wall and inclined at angles about $45^{\circ}$ to the wall. Many other events are also observed in what is a very complicated time-dependent evolution of the flow. So far, the flow has been tracked for approximately one boundary layer thickness development. Longer time sequences will be studied in the future, from which a better quantification and classification of events can be made. It is also most important to mention that when speaking of coherent structures, they must not only appear as distinctive features in a spatial sense, but they must also persist for a significant period of time during the evolution of the boundary layer.

The qualitative nature of this Mach 4 boundary layer is very similar to that observed in incompressible wall turbulence at similar Reynolds numbers. This includes the Blackburn et al. channel flow study and also the studies of Cantwell et al. (1997) and Chacin et al. (1996), who used zero-pressure-gradient turbulent boundary layer data sets. This makes sense because although the net flow is supersonic, the relative speed between convecting coherent structures is subsonic, at least for the structures observed here. This agreement between compressible and incompressible wall flows suggests that kinematic physical models for in- compressible wall turbulence, such as those described by Perry \& Chong (1982) and Perry \& Marusic (1995), can perhaps be extended to compressible flows. Fernando \& Smits (1988) also came to a similar conclusion based in part on observations of experimental flow visualizations.

\subsection{Feature Tracking}

The long term goal of our work is to develop feature extraction tools which can track the evolution of structures and provide important physical insight into the dynamical processes. At present we have developed a rudimentary region finding utility as a first step towards this goal. The technique is simple in that it employs a recursive, monotonically descending, threshold-limited flood-fill algorithm to segment individual regions associated with user-selected local maxima in the normalized level-set volume of interest at a single time step. Segmented regions can be rendered in isolation, or highlighted within the larger context of the surrounding flow through the application of a region-specific color wash during volume rendering.

Figure 4 shows an example of where the algorithm has been used to observe what appears to be a vortex connection process. Two coherent structures, identified by the discriminant level-set are volume filled with red and blue respectively. These structures are then tracked manually between time-frames. A total of 100 time-frames was available for this study and the figure shows 4 time-frames which have been chosen from this set. The first time-frame shows the structures in close proximity to each other but clearly apart. The next time-frame shown shows the structures "touching" and evidenced by the blending of color near the intersection point. Regions of purple and pink indicate a mixing of the blue and red structures. The next two frames show the structures connecting into one structure. It has long been speculated that vortex pairing and connection plays a key role in the growth of scales in turbulence. Further work is needed with automated tracking algorithms to fully explore these ideas.

Another example of an application of the technique is shown in Fig. 5. Figure 5(a) shows the visualization of level-sets of $\mathrm{S}$ where a large number of structures are seen. The structures colored red have been volume filled starting from "seeds" placed in local maxima of $S$ in planes between $0.25<z / \delta<0.35$ (where $z$ is wall-normal distance and $\delta$ is the boundary layer thickness). Figure 5(b) shows the selected structures in isolation with all other background structures masked out. With further refinement, this extraction technique will be used to look at issues such as population 
density variation per length scale of structure throughout the layer.

Future work will concentrate on resolving problem areas before a fully automated feature tracking scheme can be developed. For example, a strict enforcement of the requirement that region expansion be locally restricted to neighboring voxels of equal or lesser levelset has the potential to lead to spurious subdivision. We need to do further work to develop a system that can produce reliable results over a variety of different flow conditions with a minimum of user interaction. Other schemes will also be considered such as those proposed by Silver \& Wang (1997) where full volume overlap tests are used for the tracking. In addition to this, further work is needed for exploring alternative and new feature extraction tools. So far we have found the discriminant level-set to be the most useful.

\section{Acknowledgments}

This work is supported by the National Science Foundation grant NSF/ACI-9982274. This work is also supported by the Air Force Office of Scientific Research grant AF/F49620-98-1-0035. This work is also sponsored in part by the Army High Performance Computing Research Center under the auspices of the Department of the Army, Army Research Laboratory cooperative agreement DAAH04-95-2-0003 / contract DAAH04-95-C-0008, the content of which does not necessarily reflect the position or the policy of the government, and no official endorsement should be inferred. A portion of the computer time was provided by the University of Minnesota Supercomputing Institute.

\section{References}

3 Levoy, M. (1988). "Display of Surfaces from Volume Data", IEEE Computer Graphics and Applications, vol. 8, no. 3, May 1988, pp. 29-37.

3 Foley, V.D., Feiner and Hughes (1990). Computer Graphics: Principles and Practice, second edition in $\mathrm{C}$, Addison Wesley.

3 Silver, D., and Wang X. (1997) "Tracking and Visualizing Turbulent 3D Features," IEEE Transactions on Visualization and Computer Graphics, vol. 3, no. 2, April-June 1997, pp. 129-141.

3 Martin, M.P., and G.V. Candler, "DNS of a Mach 4 Boundary Layer with Chemical Reactions," $A I A A$ Paper No. 2000-0399, January 2000.

3 Weirs, V.G., and G.V. Candler, "Optimization of Weighted ENO Schemes for DNS of Compressible Turbulence," AIAA Paper No. 97-1940, July 1997.

3 Olejniczak, D., and G.V. Candler, "Numerical Testing of a Data-Parallel LU Relaxation Method for Compressible DNS," AIAA Paper No. 97-2133, July 1997. 


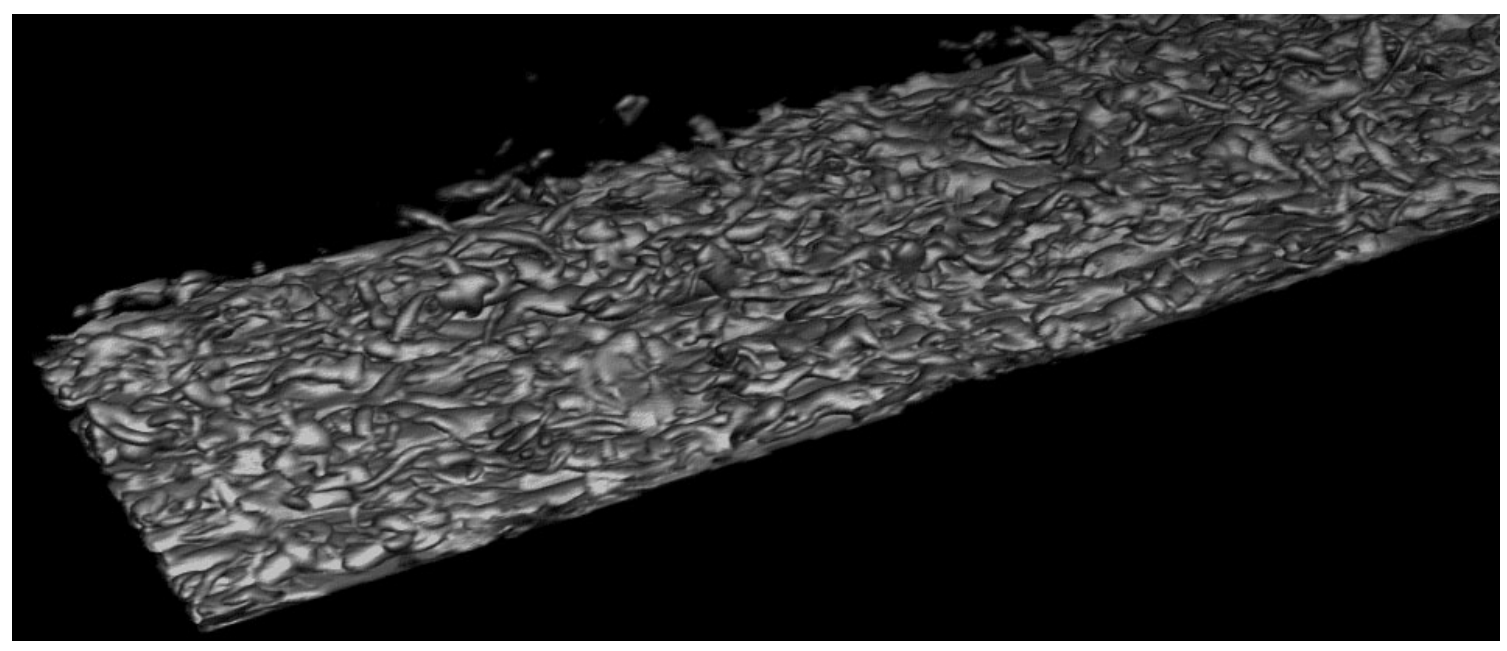

(a)

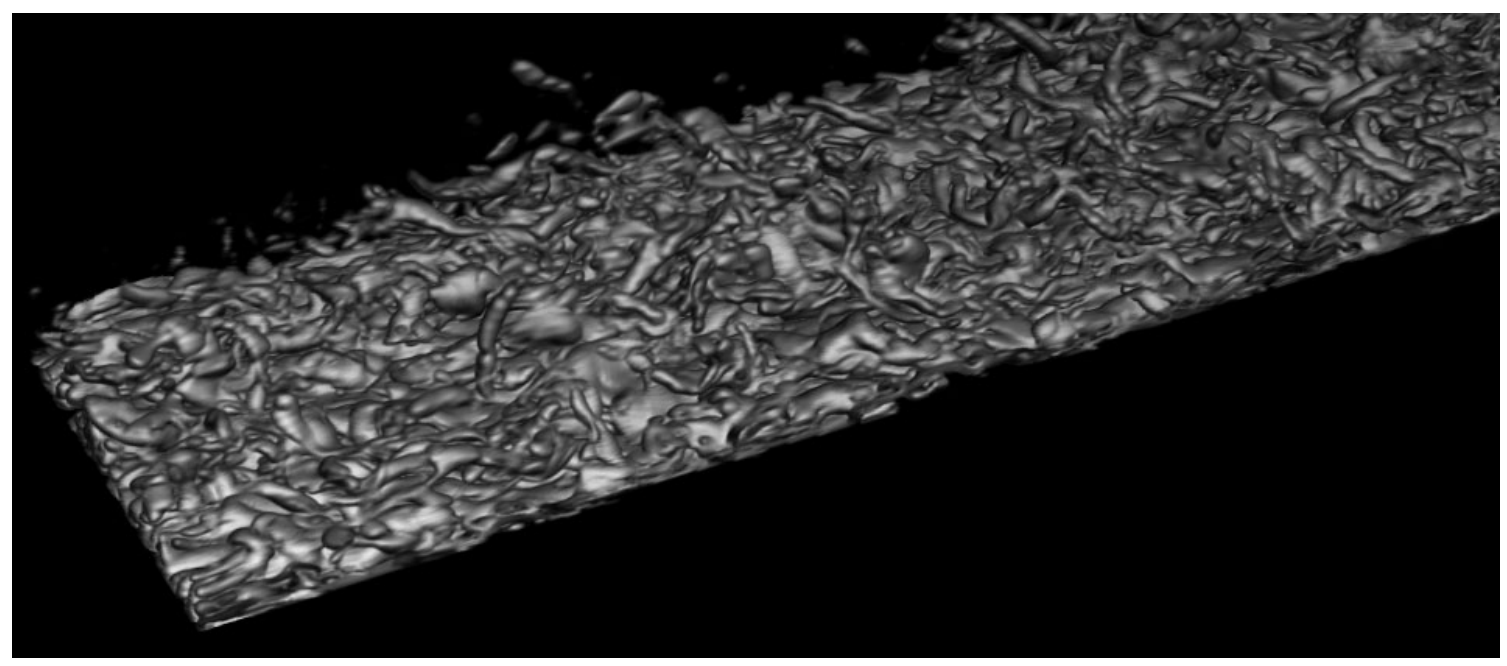

(b)

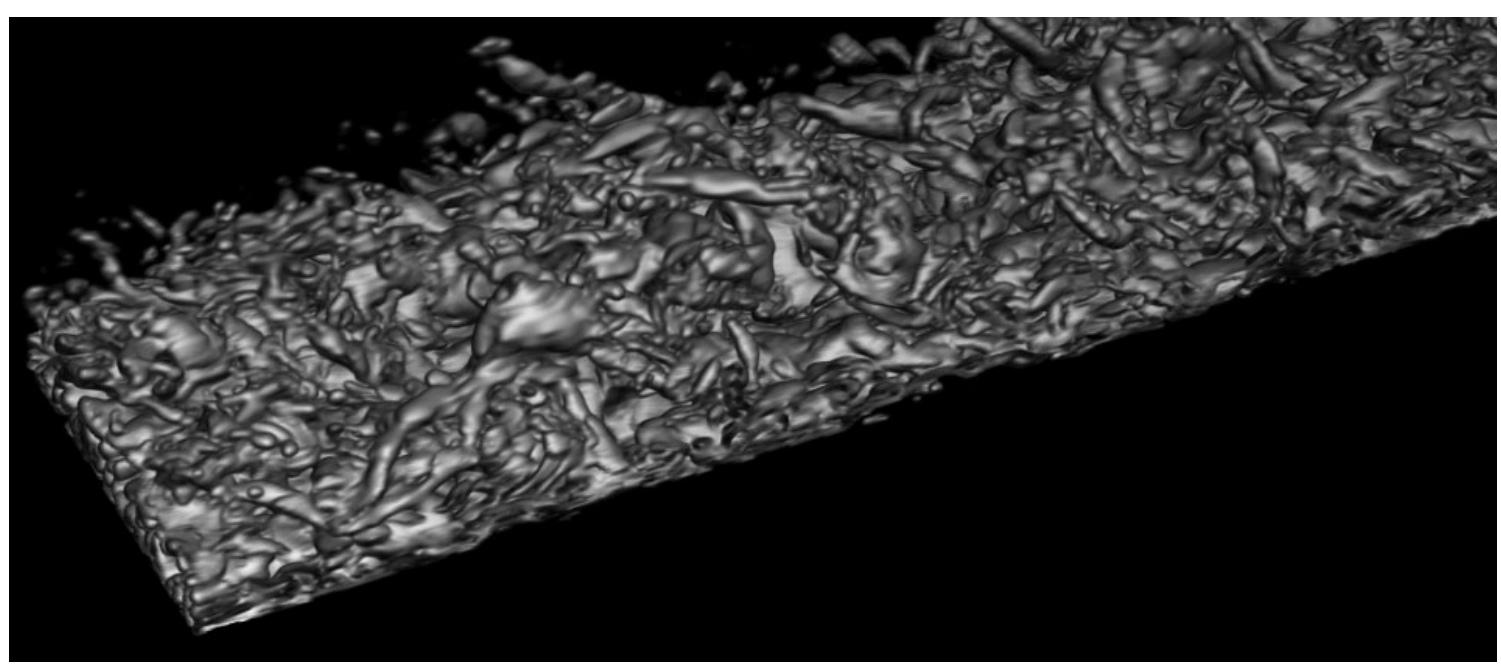

(c)

FiguRE 1. Volume rendering of level-sets of the enstrophy, $E$, in the Mach 4 turbulent boundary layer. Rendering threshold decreases from (a) to (c). 


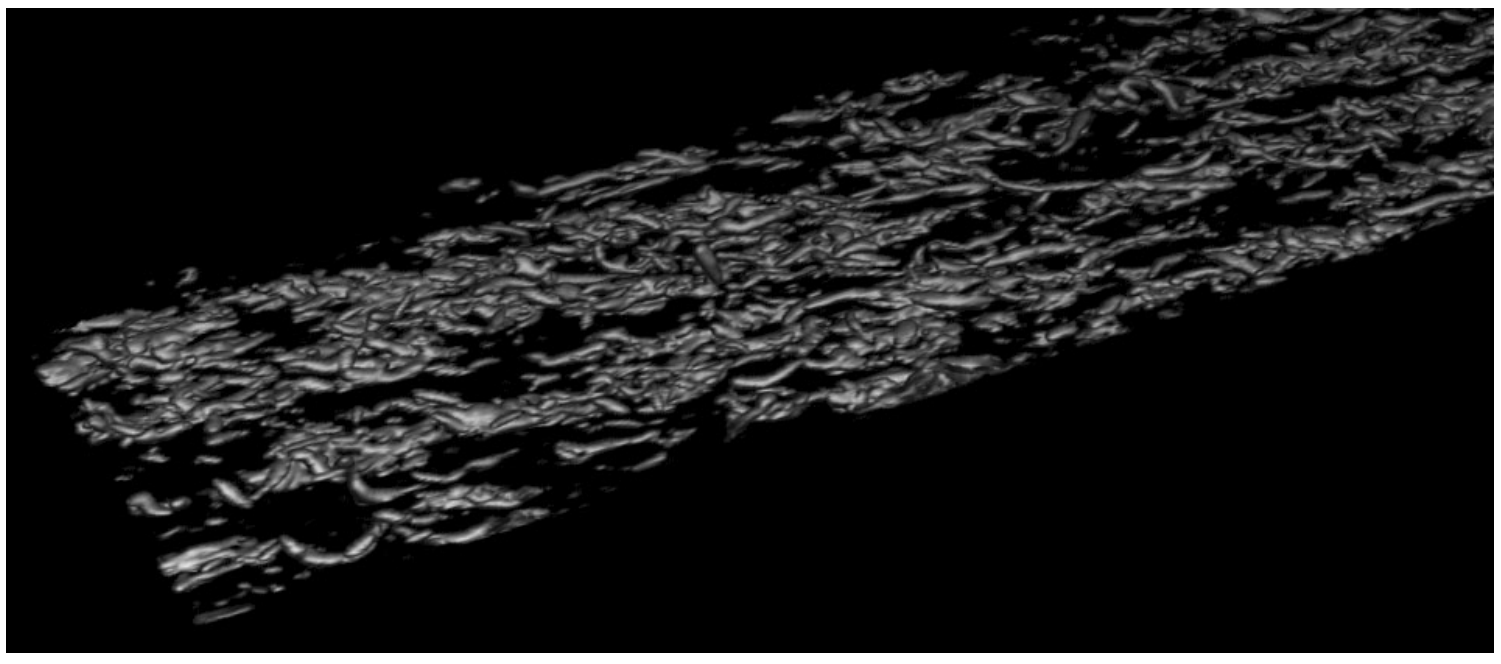

(a)

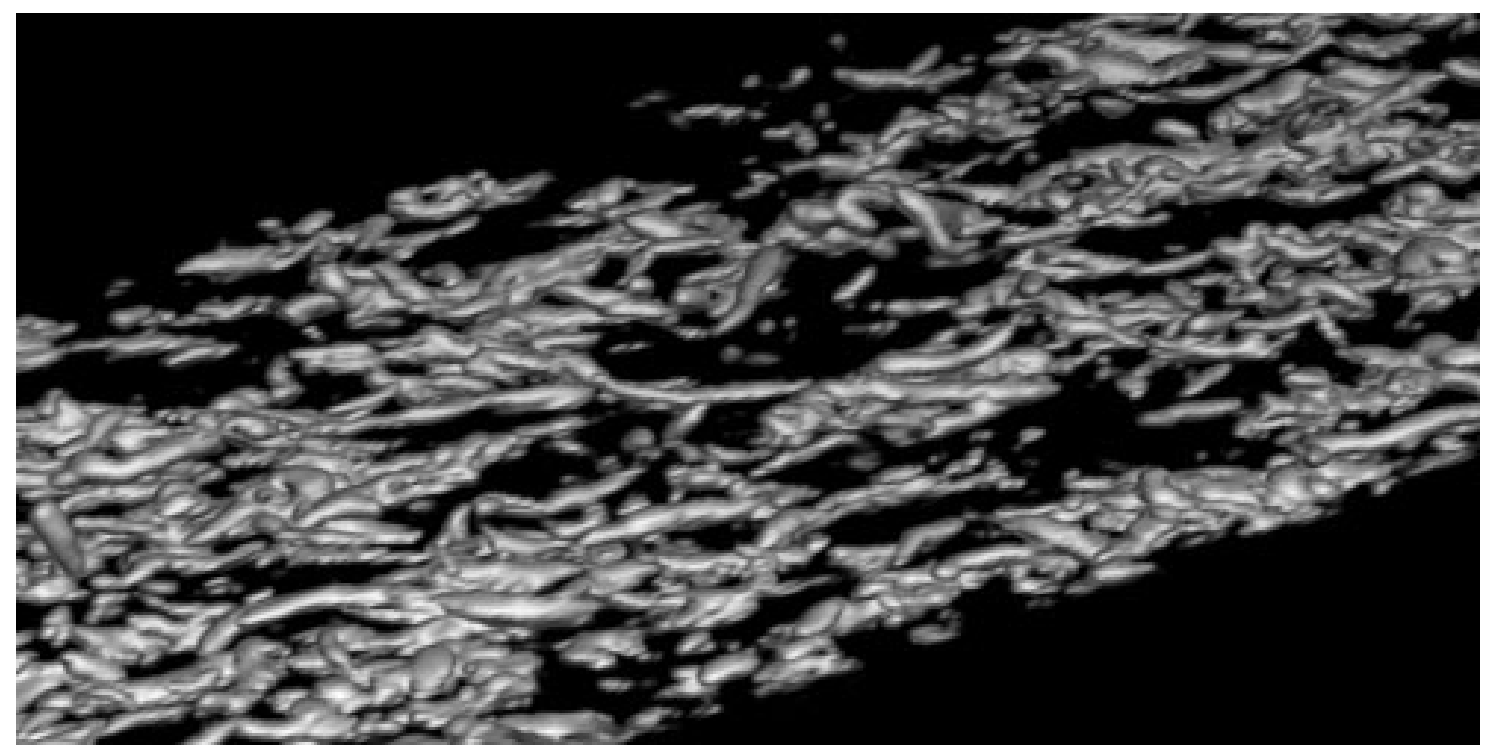

(b)

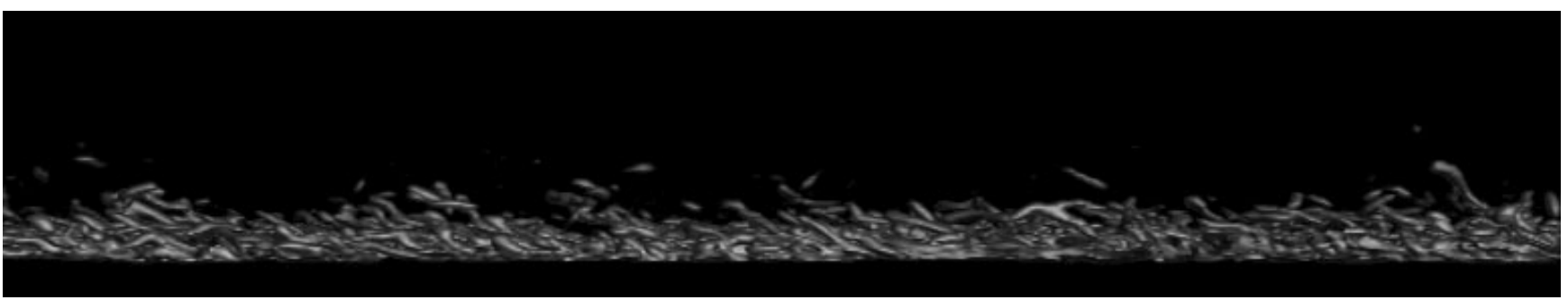

(c)

FiguRE 2. Volume rendering of level-sets of the discriminant $\Delta$, in the Mach 4 turbulent boundary layer. (b) is an enlargement of a portion of (a); (c) shows the boundary layer from the side (flow is from right to left). 


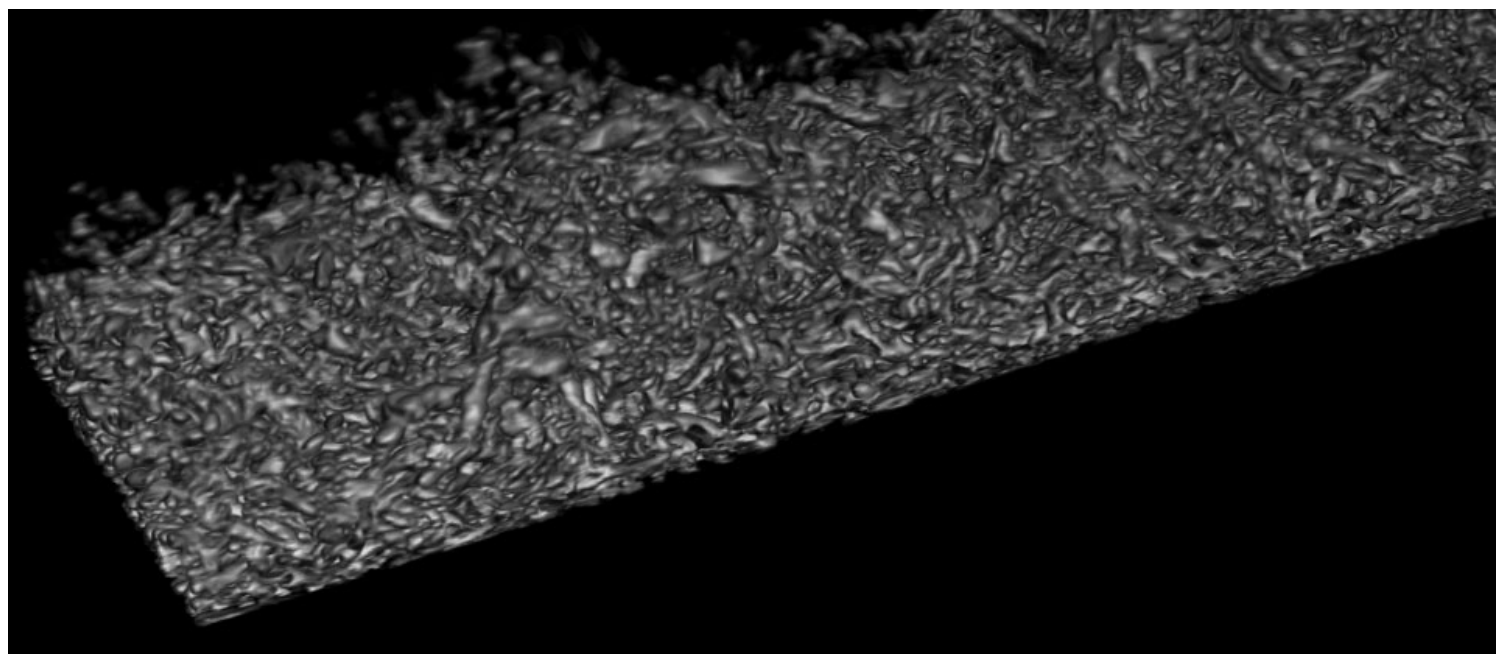

(a)

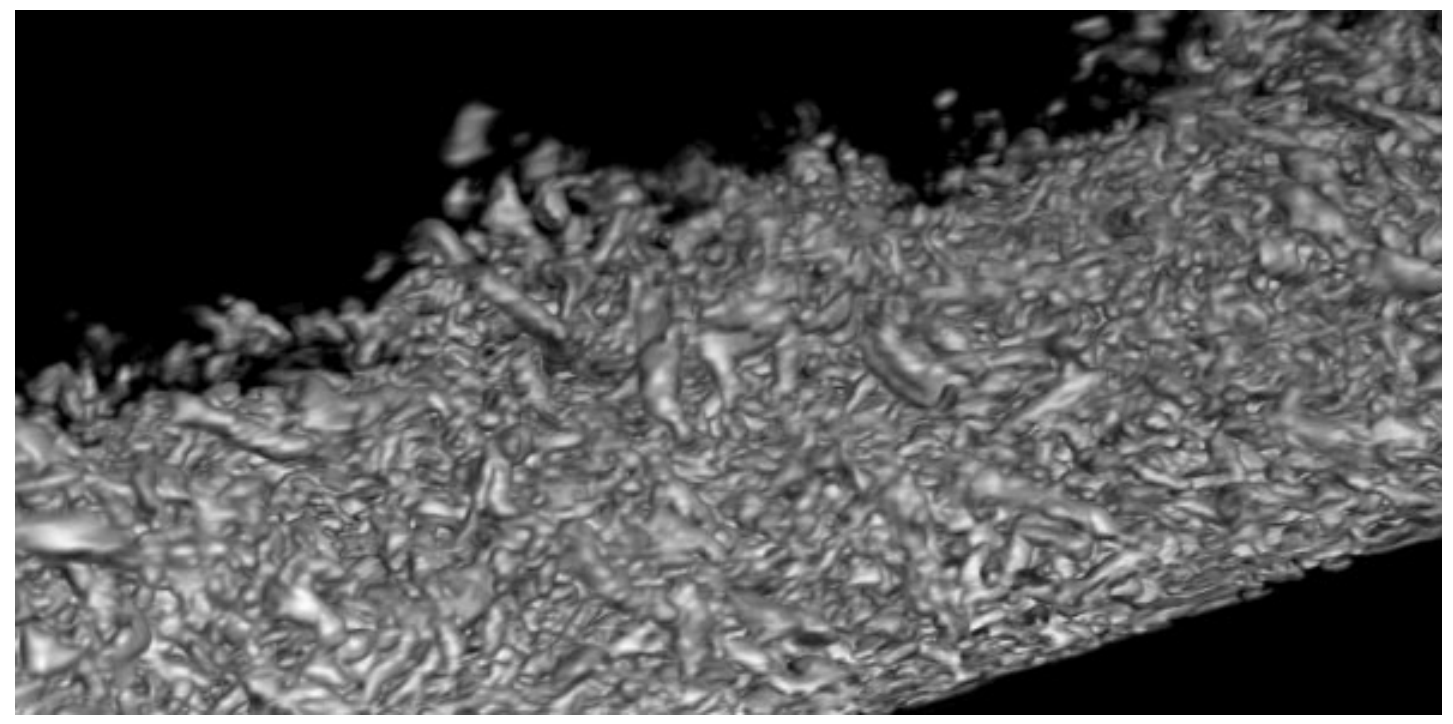

(b)

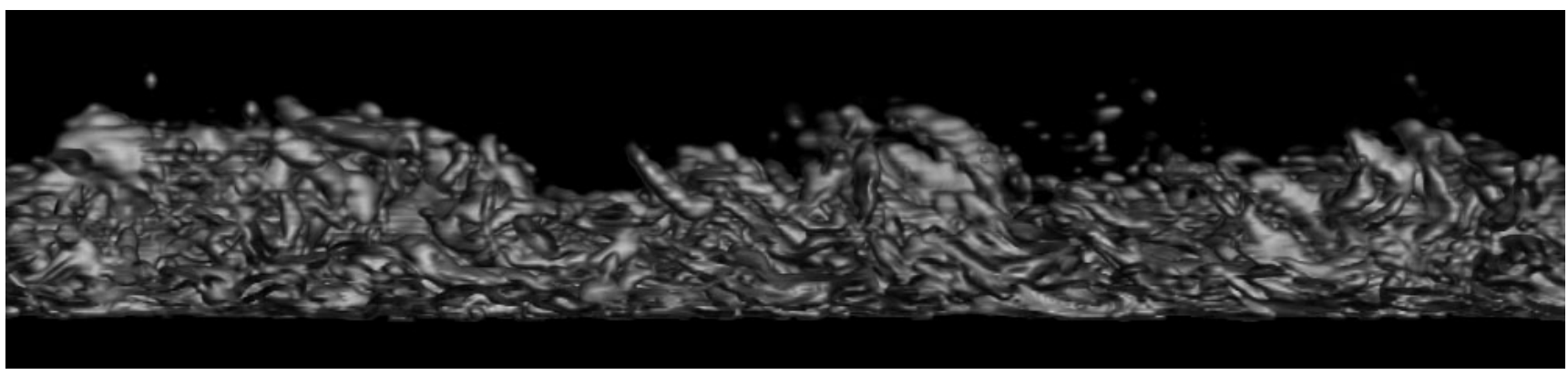

(c)

Figure 3. Volume rendering of level-sets of the swirl strenght $S$, in the Mach 4 turbulent boundary layer. (b) is an enlargement of a portion of (a); (c) shows the boundary layer from the side (flow is from right to left). 


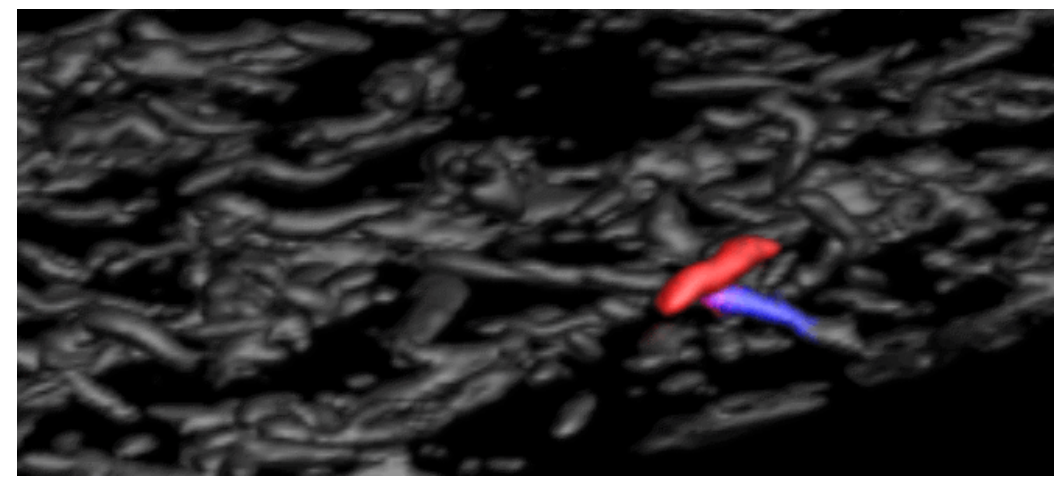

(a) Frame 29

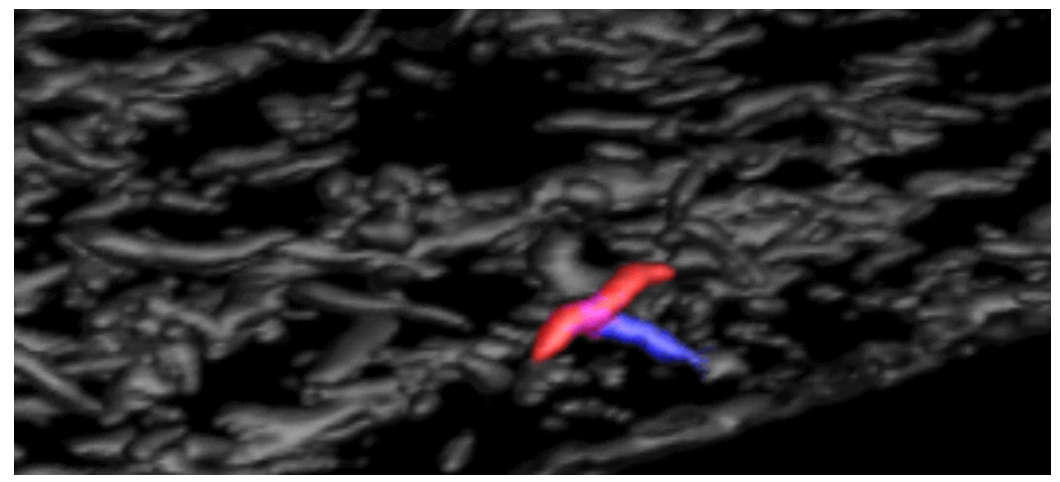

(b) Frame 56

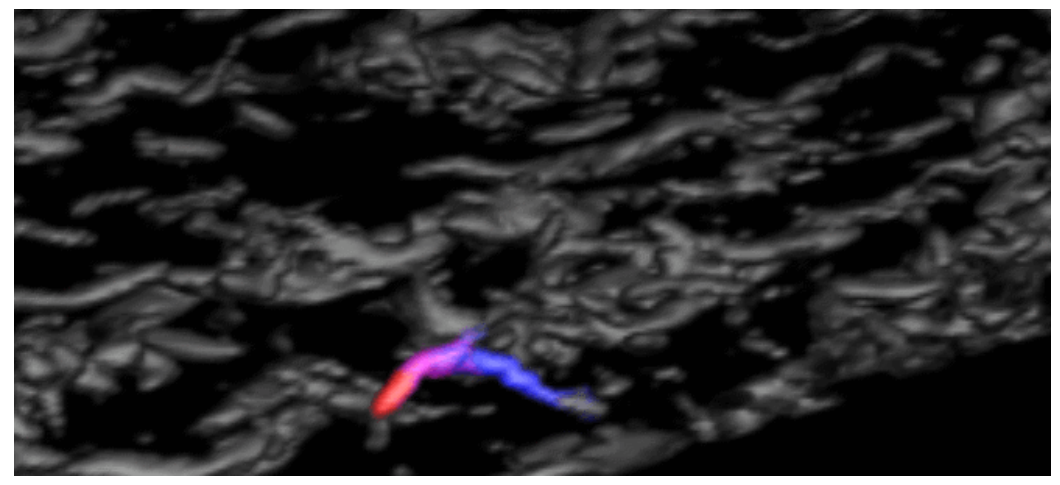

(c) Frame 84

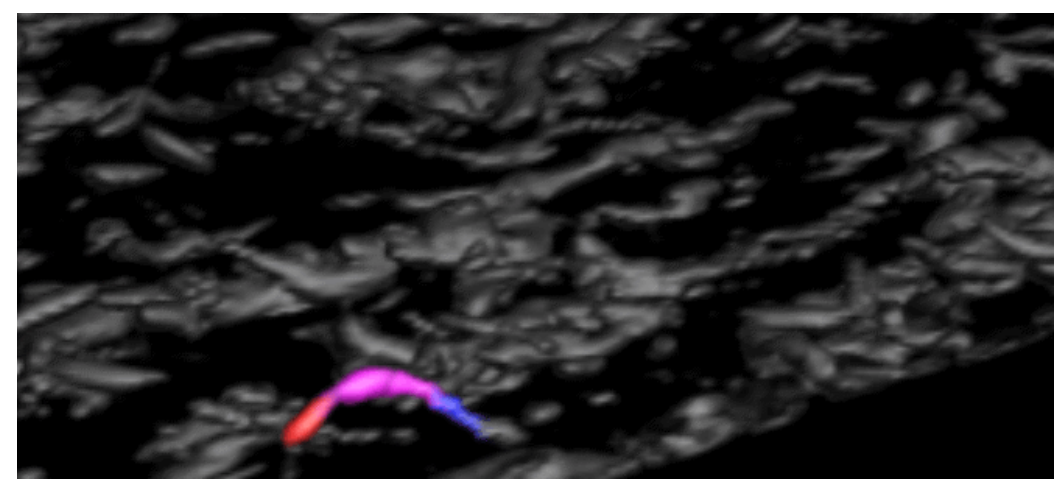

(d) Frame 100

FiguRE 4. Enlargement of volume rendering of level-sets of the discriminant $\Delta$ at different time steps showing vortex connection. Two structures have been color-filled with red and blue; purple indicates the region common to both structures. Non-dimensional time between frames: $t u_{\tau} / \delta=5.6 \times 10^{-4}$ or $t U_{e} / \delta=0.013$. 


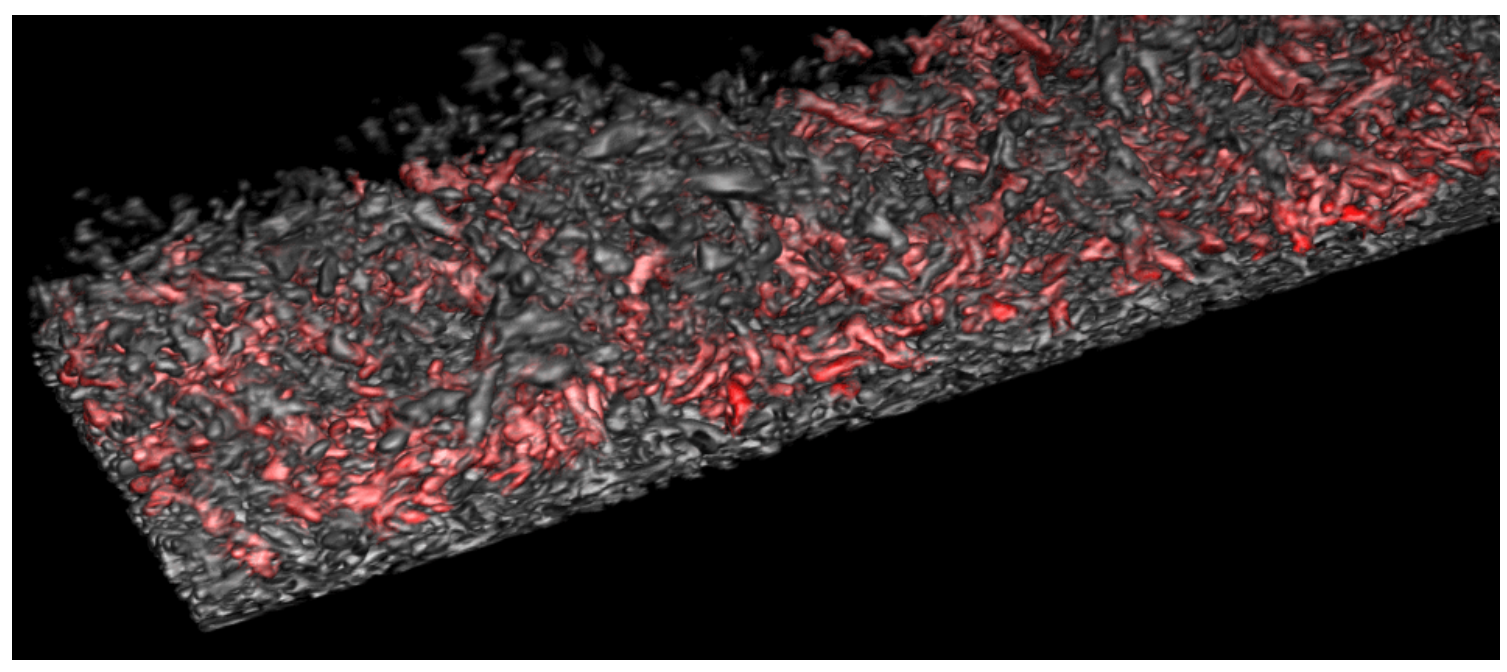

(a)

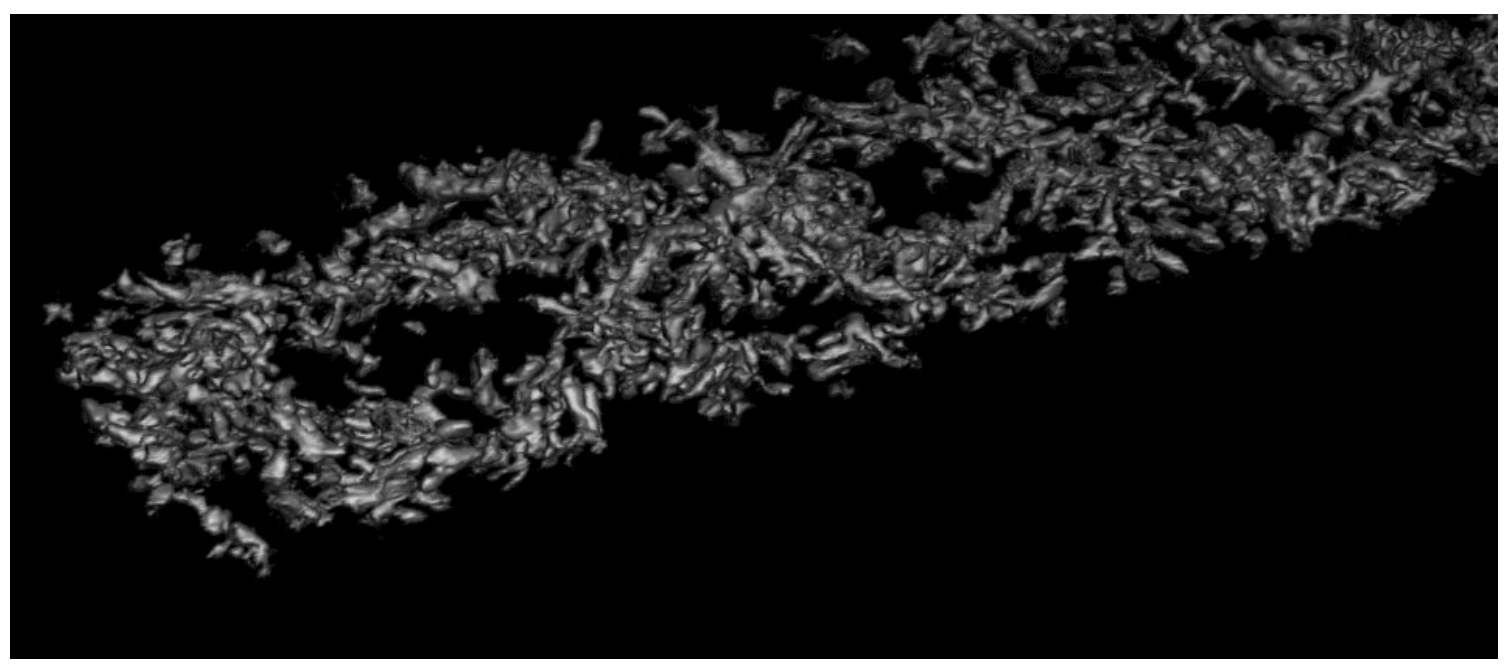

(b)

Figure 5. Volume rendering of level-sets of the swirl strenght $S$, in the Mach 4 turbulent boundary layer. The local maxima of $S$ have been used to seed the volume filling method in the $0.25 \leq z / \delta \leq 0.35$ region; the extracted structures are shown in red in (a) and separately in (b). 